Int. J. Contemp. Math. Sciences, Vol. 2, 2007, no. 26, 1271 - 1278

\title{
On the Pull-Back of Metabelian Topological Groups
}

\author{
H. Sahleh \\ Department of Mathematics \\ University of Guilan \\ P.O. Box 1914, Rasht, Iran \\ sahleh@guilan.ac.ir
}

\begin{abstract}
Let $Q$ be a topological group , $N$ a trivial $Q$-module and $(e): 0 \rightarrow$ $N \stackrel{i}{\rightarrow} \Gamma \stackrel{\pi_{i}}{\rightarrow} Q \rightarrow 0$ a topological extension with a continuous section i.e. $u: Q \rightarrow \Gamma$ such that $\pi_{i} u=I d_{Q}$. We show that if $\Gamma$ is finitely presented metabelian then the pull-back of $(e)$ is finitely presented.
\end{abstract}

Mathematics Subject Classification: 22A05

Keywords: Metablian topological group; Topological extension; Pull-back

\section{Introduction}

Let $Q$ be a topological group and $N$ a trivial $Q$-module . A topological extension of $Q$ by $N$ is a short exact sequence $0 \rightarrow N \stackrel{i}{\rightarrow} G \stackrel{\pi}{\rightarrow} Q \rightarrow 0$, where $i$ is a topological embedding onto a closed subgroup and $\pi$ an open continuous onto homomorphism. The continuous map $u: Q \rightarrow G$ is a section if $\pi u=I d_{Q}$.

All topological spaces are assumed to be Tychanov (completely regular, Hausdorff). The Free topological group is in Markov sense [9]. If $X$ is completely regular, then the (Markov) free topological group on $X$ is the group $F(X)$ equipped with the finest group topology inducing the given topology on $X$ as a subspace. Such a topology always exists [9], and has the universal property of the following kind:every continuous mapping $f$ from $X$ to an arbitrary topological group $G$ lifts to a unique continuous homomorphism

$$
g: F(X) \rightarrow G
$$

i.e.the restriction of $g$ to $X$ is $f$. For information on free topological group see $[9,8]$. 
Let $0 \rightarrow N_{i} \rightarrow \Gamma_{i} \stackrel{P_{i}}{\rightarrow} Q \rightarrow 0$ be a topological extension with a continuous section $u_{i}: Q \rightarrow \Gamma_{i}, P_{i} u_{i}=I d_{Q}$.

Let $P=\left\{\left(\gamma_{1}, \gamma_{2}\right) ; \gamma_{1} \in \Gamma_{1}, \gamma_{2} \in \Gamma_{2}, P_{1}\left(\gamma_{1}\right)=P_{2}\left(\gamma_{2}\right)\right\}$, the fiber product . Consider the case where $\Gamma_{1}=\Gamma_{2}=\Gamma, N=N_{1}=N_{2}$ and $P_{1}=P_{2}$. Then $P$ is called "untwisted".

The main question we consider is as follows: when $P$ is finitely presented? There have been some algebraic results in this direction .

If $\Gamma$ is free and $Q$ and $N$ are infinite then $P$ is never finitely presented [3,7]. On the other hand if $P_{1}=P_{2}$ and $N$ is finitely generated, $\Gamma$ is finitely presented and $Q$ is of type $F_{3}$, then $P$ is finitely presented [2]. Recall that a group $G$ is of type $F_{n}$ if there is a conncted aspherical CW complex with fundamental group isomorphic to $G$.

Inspired by [1], we consider extensions of metabelian topological groups. We show that for a topological extension , $(e): 0 \rightarrow N \rightarrow \Gamma \rightarrow Q \rightarrow 0$, if $\Gamma$ is finitely presented metablian group then the untwisted fiber product of $(e)$ is finitely presented.

In section 1 we define the pull-back in the category of topological groups. In section 2, the metabelian topological group is defined. We show that the subgroup and homomorphic image of a metabelian is again metabelian. In section 3 , the main result is proved.

\section{Pull-back of topological groups}

In this section we recall the semidirect product in the category of topological groups[10,4] and will define the pull-back of topological groups.

Definition 1.1 Let $N$ and $Q$ be topological groups. The semidirect product of $Q$ and $N$ is an exact sequence $0 \rightarrow N \stackrel{i}{\rightarrow} G \stackrel{\pi}{\rightarrow} Q \rightarrow 0$ with a continuous homomorphism $u: Q \rightarrow G$ such that $\pi u=I d_{Q}$.

Sometimes $G$ itself is called a semidirect product of $Q$ and $N$.

Examples.

(1) A direct product $N \times Q$ is a semidirect product of $N$ by $Q$ (also $Q$ by N)

(2) An abelian group is a semidirect product iff it is a direct (usually called a direct sum ) since every subgroup of an abelian group is normal.

(3) cyclic groups of prime power order are not semidirect product since they can not be direct sum of two proper subgroups. 
Two exact sequences $0 \rightarrow Y \rightarrow X \rightarrow Z \rightarrow 0$ and $0 \rightarrow Y \rightarrow X_{1} \rightarrow Z \rightarrow 0$ are said to be equivalent if there is a continuous homomorphism $T$ making the following diagram commutative:

$$
\begin{aligned}
& 0 \rightarrow Y \rightarrow X \rightarrow Z \rightarrow 0 \\
& \begin{array}{l}
\| \\
0 \rightarrow Y \\
\quad \rightarrow \quad X_{1} \rightarrow Z
\end{array} \rightarrow 0
\end{aligned}
$$

The classical 3-lemma of algebra implies that $T$ must be bijective. In the category of topological groups a 3-lemma diagram exists and $T$ is open (see [6]).

Given a topological extension, $e=(G, \pi)$, of a $Q$-module $N$ by $Q$ and a continuous homomorphism $\gamma: Q_{1} \rightarrow Q$, we define the topological extension e. $\gamma$ of $N$ by $Q$ as the top row of the following diagram:

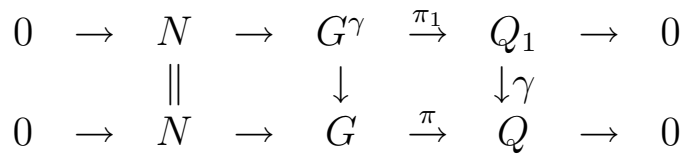

where $G^{\gamma}=\left\{(g, q) \in G \times Q_{1} ; \pi(g)=\gamma(q)\right\}$. This construction is called the pull-back of the extension $e$. Consider the case where $Q=Q_{1}, \pi_{1}=\pi$. We call the fiber product "untwisted".

Remark. If the extension $(e)$ has a continuous section then $e . \gamma$ has a section. For if $u: Q \rightarrow G$ is a map such that $\pi u=1_{Q}$ then $\delta: Q_{1} \rightarrow G^{\gamma}$ defined by $\delta(q)=(u(q), q)$ is a continuous section for $e \cdot \gamma, \pi \delta(q)=\pi(g, q)=q$ . Hence $\pi \delta=1_{Q_{1}}$.

The untwisted fiber product has a natural semidirect product decomposition.

Lemma 1.2 Let $P$ be the untwisted fiber product associated to a short exact sequence $0 \rightarrow N \rightarrow \Gamma \rightarrow Q \rightarrow 0$ with a continuous section $u: Q \rightarrow \Gamma$. Let $\hat{\Gamma}=\{(x, x) \in \Gamma \times \Gamma ; x \in \Gamma\}$ be the diagonal copy of $\Gamma$ in $\Gamma \times \Gamma$, $\Delta: \Gamma \rightarrow \Gamma \times \Gamma, \Delta(x)=(x, x)$ and let $N_{1}=N \times\{1\}$, then $P=\hat{\Gamma} \propto N_{1}$.

Proof. straightforward.

Remark. The action of $(x, x) \in \hat{\Gamma}$ on $(n, 1) \in N_{1}$ is the action of $x$ by conjugation on $N \subseteq \Gamma$.

There is a further decomposition of $P$.

Lemma 1.3 The fiber product associated to any pair of short exact sequence $0 \rightarrow N_{i} \rightarrow \Gamma_{i} \rightarrow Q \rightarrow 0, i=0,1$, has the form $0 \rightarrow N_{1} \times N_{2} \rightarrow P \rightarrow Q \rightarrow 0$.

Proof. It is clear that $N_{1} \times N_{2}$ in normal in $P$ and it is the kernel of the $\operatorname{map}\left(x_{1}, x_{2}\right) \mapsto p_{i}\left(x_{i}\right)$. 


\section{Metabelian topological groups}

In this section we will define the metabelin topological group and will show that the subgroup and the homomorphic image of a metabelian is metabelian.

Metabelian groups can be thought of as groups that are "close" to being abelian. The closeness is reflected in the structure of their commutator subgroups

Definition 2.1 A topological group $G$ is metabelian if there exists an abelian closed normal subgroup $N$ of $G$ such that $G / N$ is abelian.

For example the dihedral group is metabelian as it has a cyclic normal subgroup of index 2 .

It is clear that every abelian group is metabelian.

In fact $G$ is metabelin if the commutator, $G^{\prime}=[G, G]$, is abelian as the following result shows: .

Theorem 2.2 Let $G$ be a topological group and $H$ a closed normal subgroup of $G$. Then $G / H$ is abelian if and only if $[G, G] \subseteq H$.

Proof. Suppose $G / H$ is abelian. Let $x \in[G, G], x=a b a^{-1} b^{-1}, a, b \in G$. Now

$$
\begin{aligned}
x N & =\left(a b a^{-1} b^{-1}\right) H \\
& =(a H)(b H)\left(a^{-1} H\right)\left(b^{-1} H\right) \\
& =(b H)(a H)\left(a^{-1} H\right)\left(b^{-1}\right) \\
& =\left(b a a^{-1} b^{-1}\right) H=H
\end{aligned}
$$

So $x \in H$ Hence $[G, G] \subseteq H$. Conversely , suppose $[G, G] \subseteq H$. We show that $G / H$ is abelian. Let $a H, b H \in G / H$. By assumption $a b a^{-1} b^{-1} \in H$. Now $(a H)\left(b(H)\left(a^{-1}\right)\left(b^{-1}\right)=\left(a b a^{-1} b^{-1}\right)=H\right.$, since $H$ in normal. On the other hand $a b a^{-1} b^{-1} H=H$ because $a b a^{-1} b^{-1} \in H$. Similarly, $(b H)\left(a(H)\left(b^{-1} H\right)\left(a^{-1} H\right)=\right.$ $\left(b a b^{-1} a^{-1}\right)=H$.

Hence $(a H)\left(b(H)\left(a^{-1}\right)\left(b^{-1}\right)=(b H)\left(a(H)\left(b^{-1} H\right)\left(a^{-1} H\right)\right.\right.$.

So $(a b) H=((b a) H)\left(b^{-1} a^{-1} b a H\right)=(b a) H$.

Theorem 2.3 The group $G$ is metabelian iff $G^{\prime \prime}=1$, where $G "=\left[G^{\prime}, G^{\prime}\right]$.

Proof. Let $G$ be a metabelian topological group . By definition there is a closed abelin normal subgroup $N$ of $G$ such that $G / N$ is abelian . By theorem $2.1, G^{\prime} \subseteq N$. Since $N$ is abelian , $G^{\prime}=1$. Hence $G^{\prime \prime}=1$.

Conversely suppose $G^{\prime \prime}=1$. We will show that $G$ is metabelian. If $G$ is abelian then it is metabelian. So consider the case where $G$ is not abelian and 
hence $G " \neq 1$. First we will find a closed abelian normal subgroup of $G$.

Let $x, y \in G^{\prime}$. Since $G^{\prime \prime}=1$, then $x y x^{-1} y^{-1}=1$ and hence $x y=y x$. therefore, $G^{\prime}$ is abelian. Let $g \in G, h \in G^{\prime}$. Now $g h g^{-1} h^{-1} \in G^{\prime}$ because $g h g^{-1} h^{-1}$ is the commutation of $g, h \in G^{\prime}$. Hence $g h g^{-1}=\left(g h g^{-1} h^{-1}\right) h \in G^{\prime}$. therefore, $G^{\prime}$ is a normal subgroup of $G$. By [8], $\bar{G}^{\prime}$ is abelian and normal in $G$. By theorem 2.1, $G / \overline{G^{\prime}}$ is abelian since $G^{\prime \prime} \subseteq G^{\prime}$. Thus $G$ has an abelian closed normal subgroup $\overline{G^{\prime}}$ such that $G / \overline{G^{\prime}}$ is abelian. So $G$ is metabelian.

The category of metabelian group is closed under the homomorphic images and subgroups.

Theorem 2.4 If $H$ is a subgroup of a metabelian group $G$, then $H$ is metabelian.

Proof. Let $G$ be a metabelian group and $H$ a subgroup of $G$. By theorem $2.3, G^{\prime \prime}=1$. It is clear that $[H, H]$ is a subgroup of $G$. Since $H \subseteq G$ then for $a, b \in G a b a^{-1} b^{-1} \in G^{\prime}$. Therefore, $H^{\prime}$ is a subgroup of $G^{\prime}$. So $H^{\prime \prime}$ is a subgroup of $G$ ". Now $H^{\prime \prime}=1$ since $G "=1$. Thus by theorem $2.3, H$ is metabelian.

Theorem 2.5 The homomorphic image of a metabelian topological group is metabelian.

Proof. Let $\phi: G \rightarrow K$ be a continuous homomorphism from the metabelian group $G$ to a group $K$. We show that $\phi(G)$ is metabelian. By theorem 2.3 it is enough to show that $(\phi(G)) "=1$.

Let $\alpha \in(\phi(G))^{\prime}, \alpha=\phi(a) \phi(b) \phi(a)^{-1} \phi(b)^{-1}$ for some $a, b \in G$. Now $\alpha=$ $\phi\left(a b a^{-1} b^{-1}\right) \in \phi\left(G^{\prime}\right)$. Thus $(\phi(G))^{\prime} \subseteq \phi\left(G^{\prime}\right)$.

Let $\beta \in \phi\left(G^{\prime}\right), \beta=\phi\left(a b a^{-1} b^{-1}\right)$ for some $a, b \in G$. Since $\phi$ is a homomorphism then

$$
\beta=\phi(a) \phi(b) \phi(a)^{-1} \phi(b)^{-1} \in(\phi(G))^{\prime}
$$

Thus $\phi\left(G^{\prime}\right) \subseteq(\phi(G))^{\prime}$. Therefore, $\phi\left(G^{\prime}\right)=(\phi(G))^{\prime}$. We have $\left.(\phi G)\right)^{\prime \prime}=$ $\phi\left(G^{\prime}\right)=\phi\left(G^{\prime \prime}\right)$. Since $G$ is metabelian , $G^{\prime \prime}=1$, and $\phi(1)$ is the identity of $K$,i.e. $(\phi(G)) "=\phi(G ")=\phi(1)=1$. Thus by theorem $2.3, \phi(G)$ is metabelian.

\section{The main result}

In this section we show that for a finitely presented metabelian topological group, $\Gamma$, the associated pull-back is finitely presented. 
We need the following result:

Proposition 3.1 If $\Gamma_{1}, \Gamma_{2}$ are finitely generated and $Q$ is finitely presented , then the fiber product associated to any pair of short exact sequence $0 \rightarrow$ $N_{i} \rightarrow \Gamma_{i} \stackrel{p_{i}}{\rightarrow} Q \rightarrow 0$ is finitely generated.

Proof. Let $\rho_{1}: F_{1} \rightarrow \Gamma_{1}, \rho_{2} \rightarrow \Gamma_{2}$ be the continuous epimorphisms of finitely generated free topological groups $F_{1}, F_{2}$ onto $\Gamma_{1}, \Gamma_{2}$, respectively . Let $R_{1}, R_{2}$ be the complete inverse images under $\Gamma_{1}, \Gamma_{2}$ in $F_{1}, F_{2}$ of the kernels of the maps from $\Gamma_{1}, \Gamma_{2}$ to $Q, R_{1}=\rho_{1}^{-1}\left(k e r p_{1}\right), R_{2}=\rho_{2}{ }^{-1}\left(k \operatorname{krp}_{2}\right)$. Then $F_{1} / R_{1} \simeq F_{2} / R_{2} \simeq Q$ and since $Q$ is finitely presented $R_{1}, R_{2}$ are normal closures in $F_{1}, F_{2}$ of finite subsets $S_{1}, S_{2}$ [1]. Let $T$ be a finite generating set for $Q$ and $T_{1}, T_{3}$ be finite subsets of inverse images of $T$ under $\Gamma_{1}, \Gamma_{2}$. Then the fiber product $P$ is generated by the finite set

$$
\left\{\left(\rho_{1}\left(s_{1}\right), 1\right),\left(1, \rho_{2}\left(s_{2}\right)\right) \mid s_{1} \in S_{1}, s_{2} \in S_{2}, t_{1} \in T_{1}, t_{2} \in T_{2}, p_{1} \rho_{1}\left(t_{1}\right)=p_{2} \rho_{2}\left(t_{2}\right)\right\}
$$

Let $N \subset[\Gamma, \Gamma]$ and $1 \rightarrow N \rightarrow \Gamma \rightarrow Q \rightarrow 1$ be an extension of $Q$ by $N$ with $\Gamma$ finitely generated metablian and $P$ the associated fiber product. By $[5$,theorem B], $Q$ is finitely presented and hence by proposition $3.1, P$ is finitely generated . Now consider $1 \rightarrow \overline{[\Gamma, N]} \rightarrow \Gamma \rightarrow \Gamma / \overline{[\Gamma, N]} \rightarrow 1$ the extension of $\overline{[\Gamma, N]}$ by $\Gamma / \overline{[\Gamma, N]}$ and let $\tilde{P}$ be the fiber product of this extension.

We compare $P$ with $\tilde{P}$.

Lemma 3.2 $\tilde{P}$ is a normal subgroup of $P$ and the quotient is finitely generated abelian group.

Proof. By lemma 1.2 there are semi-direct product decompositions $P=$ $\Gamma \propto N$ and $\tilde{P}=\Gamma \propto \overline{[\Gamma, N]}$. The natural inclusion is that implicit in the notation. So $\tilde{P}$ is normal in $P$ and the quotient is naturally isomorphic to $N / \overline{[\Gamma, N]}$, which is abelian. This quotient is finitely generated since $P$ is.

Note: By lemma 3.2 ,to prove the main result, there is no loss of generality if we replace $N$ by $\overline{[\Gamma, N]} \subseteq \overline{[\Gamma, \Gamma]}$ and prove the theorem in that context. Since In that case, by above lemma, $P$ is an extension of the finitely presented group $\tilde{P}$ by the finitely presented group $P / \tilde{P}$ and so is itself finitely presented.

Remark 3.3. A continuous homomorphism $\nu: Q \rightarrow R$ is called a valuation of $Q$. Let $Q_{\nu}=\{q \in Q \mid \nu(q) \geq 0\}$.

The module $A$ is said to be tame if for any valuation $\nu$ of $Q$ either $A$ is finitely generated as $Q_{\nu}$-module or else it is finitely generated as a $Q_{-\nu}$-module.

Submodules of a tame module $A$ and direct product of a finite number of copies of $A$ are tame [5,proposition 2.5]. For more on "tame" see [5]. 
We need theorem $(\mathrm{A}(\mathrm{ii}))$ of $[5]$.

Theorem $3.4[5, \mathrm{~A}(\mathrm{ii})] \quad$ Consider a short exact sequence $1 \rightarrow A \rightarrow \Gamma \rightarrow$ $Q \rightarrow 1$ with $A$ and $Q$ abelian and $\Gamma$ finitely generated. Then $\Gamma$ is finitely generated iff $A$ is tame as a $Q$-module.

Theorem 3.5 If $\Gamma$ is finitely presented metabelian topological group then untwisted product associated to any short exact sequence $1 \rightarrow N \rightarrow \Gamma \rightarrow Q \rightarrow 1$ is finitely presented.

Proof. Let $\Gamma^{\prime}=\overline{[\Gamma, \Gamma]}$ and $\Gamma_{a b}=\Gamma / \overline{[\Gamma, \Gamma]}$. By lemma 1.2 we may assume $N \subset \overline{[\Gamma, \Gamma]}$, the closure of the commutator subgroup of $\Gamma$. We have a short exact sequence $1 \rightarrow \Gamma^{\prime} \propto N \rightarrow \Gamma \propto N \rightarrow \Gamma_{a b} \rightarrow 1$ where $\Gamma \propto N$ is the decomposition of $P$ given in lemma 1.2 and the inclusion of the first group is obvious. But now since we are assuming $N \subset \overline{[\Gamma, \Gamma]}$ which is abelian, the first term in this sequence is actually a direct product. Since $\Gamma$ is finitely presented , $\Gamma^{\prime}$ is a tame module over $\Gamma_{a b}$ by the "only if" part of theorem 3.4. By [lemma $7,3], \Gamma^{\prime} \times N$ is a tame $\Gamma^{\prime}$-module and the action implicit in the above short exact sequence is indeed the product action of $\Gamma_{a b}$ on $\Gamma^{\prime} \times N$. Thus by the "if" part of theorem 3.4, we are done.

\section{References}

[1] G. Baumslag, M.R. Bridson, D.F. Holt, C.F. Miller III , Finite presentation of fiber product of metablian groups, J. Pure Appl. Algebra., 181 (2003), 15-22.

[2] G. Baumslag and J.E.Roseblade, Subgroups of direct product of two free groups, J. London Math. Soc., 30 (1984), 44-52.

[3] G. Baumslag, M.R. Bridson, C.F. Miller and H.B. Short, Fiber product, non-positive curvature, and decision problems, Comment. Math. Helv., 75 (2000), 457-477.

[4] O. Berndt, A caregorical definition of semidirect products, Applied Categorical Structures. 6 (1998), 37-62.

[5] R. Bieri, R. Strebel, Valuations and finitely presented abelian groups, Proc. London Math. Soc., 41 (1980), 439-464.

[6] J.M.F. Castillo, On the "three-space" problem for locally quasi-convex topological groups, Arch. Math. 74 (2000), 253-262. 
[7] F. Grunewald, On some groups which cannot be finitely presented, J. London Math. Soc., 17 (1978), 427-436.

[8] E. Hewitt, K.A. Ross, Abstract Harmonic Analysis I, Academic Press, New York, 1963.

[9] A.A. Markov, On free topological groups, Amer. Math. Soc. Transl., 30 (1950), 11-88.

[10] H. Sahleh, The semidirect product and the first cohomology of topological groups, Int. Math. Forum, 2(28) (2007), 1363-1373.

\section{Received: May 6, 2007}

